\title{
LEP Higgs boson searches beyond the standard model and minimum supersymmetric standard model
}

\author{
PAULINE GAGNON
}

Indiana University, Bloomington, USA

Corresponding address: CERN EP, Geneva 23, CH-1211, Switzerland

\begin{abstract}
Ever since the center-of-mass energy was increased in 1995 above the $Z^{0}$ resonance, the four LEP experiments (ALEPH, DELPHI, OPAL and L3) have renewed their effort to search for the Higgs boson. Data taking ended in the year 2000 with about $130 \mathrm{pb}^{-1}$ of data collected per experiment above $206 \mathrm{GeV}$ in $e^{+} e^{-}$collisions but the data analysis is still very active. Most recently, the wealth of theoretical models and predictions has stimulated new analyses and model interpretations which go beyond the standard model and minimal supersymmetric standard model. These include the searches for charged Higgs bosons, models with two Higgs field doublets, searches for 'fermiophobic' Higgs decay, invisible Higgs boson decays, decay-mode independent searches, and limits on Yukawa and anomalous Higgs couplings. I review the searches done by the four LEP experiments and present the LEP combined results when they exist.
\end{abstract}

\section{Introduction}

The standard model (SM) predicts only one complex Higgs scalar field doublet which gives rise to one physical state, $H^{0}$. The simplest extension is given by two Higgs doublet models (2HDM) which yield five physical states: two CP-even, $H^{0}$ and $h^{0}$, one CP-odd, $A^{0}$, and two charged states, $H^{ \pm}$. The $H^{0}$ state is heavier than $A^{0}$ and $h^{0}$ and is not expected at LEP energies. Higgs triplet models predict two doubly-charged Higgs bosons, $H^{ \pm \pm}$.

At LEP, Higgs bosons could have been produced in three different ways shown in figure 1: the Higgsstrahlung process, the associated production and through a Yukawa coupling. The Yukawa mechanism is discussed in $\S 4$. The $Z^{0} h^{0}$ and $h^{0} A^{0}$ production cross-sections can be expressed in terms of the SM production crosssection, $\sigma_{\mathrm{SM}}$, and depend on two angles: $\tan \beta=v_{2} / v_{1}$, the ratio of the vacuum expectation values, and $\alpha$, the mixing angle between the two mass doublets, namely:

$$
\sigma_{Z h}=\sin ^{2}(\beta-\alpha) \sigma_{\mathrm{SM}} \quad \text { and } \quad \sigma_{A h}=\cos ^{2}(\beta-\alpha) \bar{\lambda} \sigma_{\mathrm{SM}},
$$

where $\bar{\lambda}$ accounts for $P$-wave suppression near threshold. The decay branching ratios (BR) also depend on these two angles. 


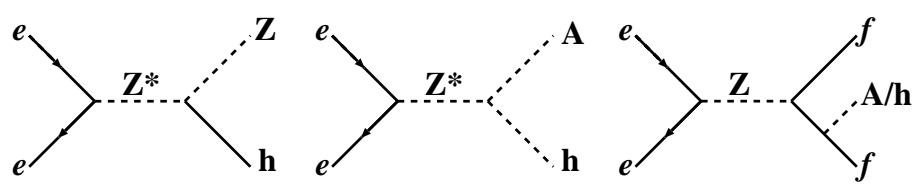

Figure 1. The three possible production mechanisms for a Higgs boson at LEP: Higgsstrahlung, pair production and through a Yukawa coupling.

The 2HD models come in two types: in Type I models, one doublet couples to fermions, the other to bosons. In Type II models, one doublet couples to uptype fermions, the other to down-types. One could also have a model where a doublet only couples to leptons, the other to quarks. The minimum supersymmetric standard model (MSSM) is a special case of 2HDM of Type II. Depending on the values of $\alpha$ and $\beta$, a specific production and decay mode can be enhanced or suppressed in eq. (1), hence the need to investigate all possibilities laid out by various theoretical scenarios. There is no way to tell if the LEP experiments have searched in every possible way for the Higgs boson. One can simply state the many searches done so far as shown in table 1. The table summarizes all final, preliminary and new LEP Higgs searches done in theoretical frameworks beyond the SM and MSSM and reviewed in this paper. Details cannot be provided here but the interested reader will find all references to individual analyses and to the existing LEP combinations. For a review of SM and MSSM Higgs searches at LEP, see Peter Igo-Kemenes' summary in these proceedings.

\section{Fermiophobic Higgs boson decays}

In the SM, Higgs boson decays to two photons are allowed but the BR is of $\mathcal{O}\left(10^{-3}\right)$. In $2 \mathrm{HDM}$ of Type I, they dominate at low Higgs mass. The fermiophobic benchmark scenario is obtained by setting all SM Higgs BR to fermions to zero, keeping the bosons relative $\mathrm{BR}$ unchanged. This gives $100 \%$ decays into photons below $m_{\mathrm{h}} \approx$ $60 \mathrm{GeV} / \mathrm{c}^{2}$, with decays into a $W^{+} W^{-}$dominating above $90 \mathrm{GeV} / \mathrm{c}^{2}$. All LEP experiments have searched for an excess of events in the $h^{0} Z^{0}$ channel with $h^{0} \rightarrow \gamma \gamma$. The $95 \%$ CL limit on the mass of a fermiophobic Higgs boson is placed at 109.7 $\mathrm{GeV} / \mathrm{c}^{2}$. In addition, the OPAL Collaboration performed a more general search for $e^{+} e^{-} \rightarrow X Y$ with $X$ a scalar decaying into $\gamma \gamma$ whereas the L3 Collaboration started looking at the $h^{0} \rightarrow W W$ and $Z Z$ channels. All details can be found in [1].

\section{Invisible Higgs boson decays}

Invisible Higgs boson decays denote decays into neutralinos $h^{0} \rightarrow \chi^{0} \chi^{0}$, which escape undetected. They are allowed in Majorona and supersymmetric models depending on the parameters choice in eq. (1). An invisibly decaying Higgs boson would be detected from the presence of the accompanying $Z$ boson decay products, either as acoplanar jets or dileptons recoiling against missing energy. The four LEP 
Table 1. Status of all individual and LEP combined searches beyond SM and MSSM models. Analyses are marked as 'final' when published, 'prel' for preliminary results, and 'new' when work has just started and no results are yet available.

\begin{tabular}{llllll}
\hline Search & ALEPH & DELPHI & OPAL & L3 & LEP \\
\hline$h^{0} \rightarrow \gamma \gamma$ & final & final & final & final & final \\
$h^{0} \rightarrow W W, Z Z$ & new & new & & final & \\
$h^{0} \rightarrow \chi^{0} \chi^{0}$ & final & prel & prel & prel & prel \\
Yukawa couplings & & prel & final & & \\
$H^{+} \rightarrow c \bar{s}, \tau \nu$ & final & prel & prel & prel & prel \\
$H^{+} \rightarrow W^{ \pm} A^{0}$ & & prel & & & \\
$H^{ \pm \pm}$ & & final & final & & \\
Flavour-indep. & final & prel & prel & prel & prel \\
Decay mode-indep. & & & final & & \\
Low $m_{\mathrm{A}}$ & & & final & & \\
Anomalous coupling & & & & prel & \\
2 HDM & & & prel & & \\
\hline
\end{tabular}

Table 2. Inputs from the four LEP experiments used for the combination in the search for invisible Higgs boson decays $\left(m_{\mathrm{H}}=110 \mathrm{GeV} / \mathrm{c}^{2}\right.$ when relevant). The data is for $E_{\mathrm{CM}}=200-209 \mathrm{GeV}$ but the limits are derived including all data.

\begin{tabular}{lllll}
\hline Experiment & ALEPH & DELPHI & L3 & OPAL \\
\hline $\mathcal{L}_{\text {int }}\left(\mathrm{pb}^{-1}\right)$ : & 215.6 & 225.1 & 217.3 & 210 \\
$\quad$ Backg. predicted/evts obs. & & & & \\
$\quad$ Acoplanar jets & $8.17 / 8$ & $35.9 / 30$ & $56.2 / 50$ & $61.8 / 47$ \\
$\quad$ Acoplanar leptons & $6.7 / 7$ & $21.7 / 13$ & $5.9 / 9$ & - \\
Total events & $14.9 / 15$ & $57.6 / 43$ & $62.1 / 59$ & $61.8 / 47$ \\
Median 95\% CL $\left(\mathrm{GeV} / \mathrm{c}^{2}\right)$ & $112.6 / 111.8$ & $110.7 / 110.7$ & $110.2 / 110.1$ & $107.4 / 108.5$ \\
Observed 95\% CL $\left(\mathrm{GeV} / \mathrm{c}^{2}\right)$ & $114.1 / 113.1$ & $113.0 / 113.0$ & $107.6 / 107.5$ & $107.0 / 107.4$ \\
\hline
\end{tabular}

experiments have searched for such decays and placed a limit of $114.4 \mathrm{GeV} / \mathrm{c}^{2}$ on the mass of an invisibly decaying Higgs boson. Individual results are summarized in table 2 and all details are given in [2].

\section{Yukawa couplings}

The Higgsstrahlung production mechanism of figure 1 can be suppressed in general 2HD models for an appropriate parameters choice in eq. (1). If pair production is also impossible (i.e. below the mass threshold), then the Yukawa production mode dominates. Its production cross-section depends on the fermion mass and an enhancement factor, $\xi_{f}, f$ being the flavour of the radiating fermion. Hence, depending on $\xi_{f}$, the Higgs would preferentially radiate from a heavy up-type or 

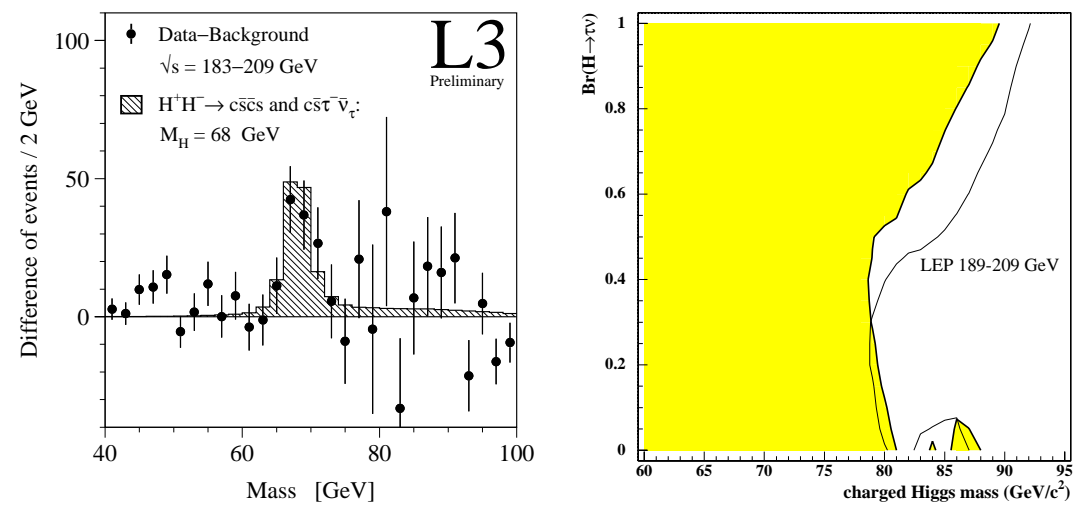

Figure 2. Left: Excess in the number of selected events in the L3 data after background subtraction. Right: The LEP combined mass limit for a charged Higgs boson.

down-type quark. DELPHI and OPAL have both searched for $e^{+} e^{-} \rightarrow b \bar{b} A^{0} / h^{0}$ with $A^{0} / h^{0} \rightarrow \tau^{+} \tau^{-}$. DELPHI has also searched for $A^{0} / h^{0} \rightarrow b \bar{b}$. Both put limits on $\xi_{d}$ (roughly less than 12) as a function of $m_{\mathrm{A}}$ and $m_{\mathrm{h}}$ as given in [3].

\section{Charged and doubly-charged Higgs boson searches}

All LEP experiments have searched for charged Higgs boson with $H^{ \pm} \rightarrow c \bar{s}$ and $\tau \nu$. The L3 experiment saw an excess around $m_{H^{ \pm}}=70 \mathrm{GeV} / \mathrm{c}^{2}$ as shown in figure 2. The other experiments observed no such excess even after emulating the L3 analysis. Hence, the L3 excess is interpreted as a $4.2 \sigma$ background fluctuation. The combined LEP mass limit is $m_{H^{ \pm}}>78.6 \mathrm{GeV} / \mathrm{c}^{2}$ at $95 \% \mathrm{CL}$, as shown in figure 2 . The DELPHI group has also recently presented results on $H^{ \pm} \rightarrow W^{ \pm} A^{0}$. Details are provided in [4].

$H^{ \pm \pm}$are predicted in Higgs triplet models. They would be pair-produced and would only decay into charged leptons and gauge bosons. They occur naturally in left-right symmetric models. Such models predict a small neutrino mass, hence their appeal. Since these models do not conserve baryon and lepton numbers, one possible decay is $H^{ \pm \pm} \rightarrow \ell^{ \pm} \ell^{ \pm}$. Both DELPHI and OPAL have searched this channel and placed limits $m_{H^{ \pm \pm}}$at 99.6 and $98.5 \mathrm{GeV} / \mathrm{c}^{2}$, respectively, for a Yukawa coupling $h_{\tau \tau}>10^{-7}$. DELPHI also has a mass limit of $99.3 \mathrm{GeV} / \mathrm{c}^{2}$ for $h_{\tau \tau}<10^{-9}$. Details are provided in [5].

\section{Flavour-blind, decay-mode independent and light CP-odd Higgs boson searches}

The Higgs boson could have escaped detection if it did not decay into $b \bar{b}$ as predicted by the SM. This coupling could be suppressed in 2HDM, MSSM and composite models for specific parameters choice. Hence, all LEP SM searches in the ZH channel with $H^{0} \rightarrow b \bar{b}$ were repeated for $H^{0} \rightarrow q \bar{q}$, allowing for any jet flavour by simply removing the $b$-tag requirements. For the four-jet channel, additional 


\section{OPAL}
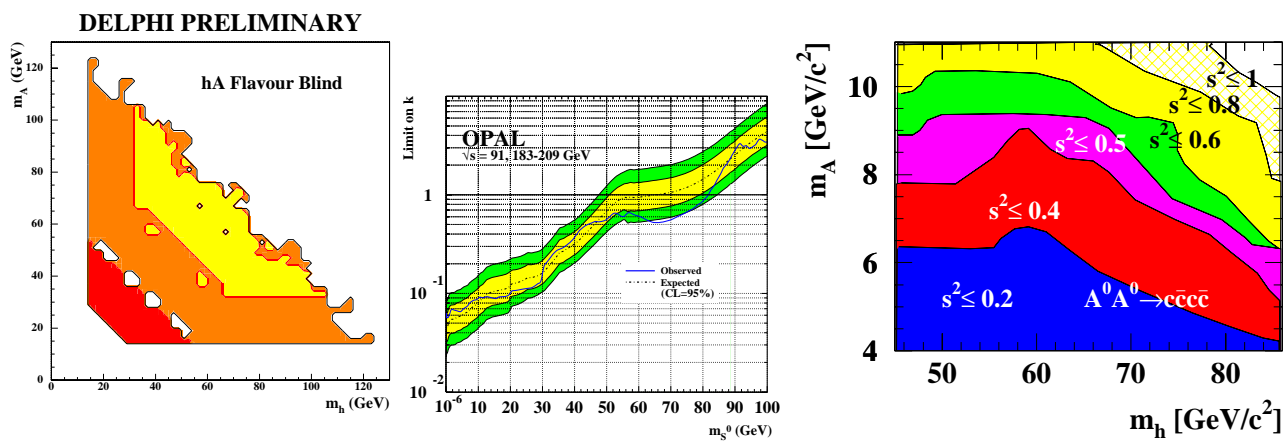

Figure 3. Left: Excluded $m_{\mathrm{A}}-m_{\mathrm{h}}$ region from the DELPHI data in the flavour-blind $h^{0} A^{0}$ channel. OPAL limits on $k$ and $s^{2}$, both being the ratio of the production cross-section with respect to the SM cross-section for, center: a decay-mode independent Higgs boson and, right: a low mass CP-odd Higgs boson with $A^{0} A^{0} \rightarrow c \bar{c} c \bar{c}$.

kinematic constraints on the $Z$ mass have been added to reduce the combinatorial background. The limit on the mass of a flavour-blind Higgs is placed at 112.9 $\mathrm{GeV} / \mathrm{c}^{2}$ for the LEP experiments combined. DELPHI has also pioneered work on the $h^{0} A^{0}$ channel, as shown in figure 3 . Details on the individual analyses and the LEP combination are given in [6].

The OPAL Collaboration has also performed a simple topological search where no assumptions are made on the decay of the Higgs boson. Events are searched for by looking at the recoil mass spectrum of the $Z^{0} \rightarrow e^{+} e^{-}$or $\mu^{+} \mu^{-}$. All data from LEP I and LEP II have been used. Limits on the production cross-section are shown in figure 3 in terms of the factor $k$, the ratio of the production cross-section to that expected in the SM. Analysis details and limits on the parameters of the stealthy-Higgs models can be found in [7].

Up to very recently, a small region of the parameter space for the MSSM nomixing scenario was left unexcluded by LEP for $m_{\mathrm{A}}$ below the $b \bar{b}$ threshold since most analyses required Higgs bosons to decay into $b \bar{b}$. The OPAL Collaboration has just ruled out a light CP-odd Higgs boson mass in the range $m_{\mathrm{A}} \simeq 2-10$ for $m_{\mathrm{h}}=45-82 \mathrm{GeV} / \mathrm{c}^{2}$. Model-independent cross-section limits are provided in [8] with an example shown in figure 3 .

\section{Anomalous Higgs boson couplings and 2HDM interpretations}

The L3 Collaboration explored the possibility of enhancement in the following channels: $e^{+} e^{-} \rightarrow H^{0} \gamma, e^{+} e^{-} H^{0}$ and $H^{0} Z^{0}$ due to unexpectedly large couplings. Limits are placed on the anomalous couplings $d, d_{B}, \Delta g_{1}^{Z}, \Delta \kappa_{\gamma}, \delta_{Z}$ in [9].

Once all LEP MSSM searches are finalized, they will be re-interpreted in the more general framework of $2 \mathrm{HD}$ models as pioneered by OPAL, exploring the entire parameter space for $\left(m_{\mathrm{A}}, m_{\mathrm{h}}, \tan \beta, \alpha\right)$. Preliminary limits on excluded $m_{\mathrm{A}}-m_{\mathrm{h}}$ regions by the OPAL data for different values of $\alpha$ and $\tan \beta$ are provided in [10]. 


\section{Conclusions}

If the Higgs boson has not been observed at LEP, it is not for lack of trying! Many scenarios have been explored and new efforts are still underway as shown in table 1 . Hopefully, the ongoing efforts at the TeVatron and the LHC will soon be rewarding, yielding either the Higgs boson itself or at least a slew of new particles.

\section{References}

[1] ALEPH: Phys. Lett. B487, 241 (2000) DELPHI: Phys. Lett. B507, 89 (2001) L3: CERN-EP/2002-080 and Phys. Lett. B534, 28 (2002) OPAL: Phys. Lett. B544, 44 (2002) LEP combination: LHWG Note/2002-02

[2] ALEPH: Phys. Lett. B526, 191 (2002) DELPHI: 2002-025-CONF-559 (ICHEP 2002) L3: Note \#2690 (EPS 2001) OPAL: Physics Note \#472 (ICHEP 2002) LEP combination: hep-ex/0107032

[3] DELPHI 2002-037-CONF-571 (ICHEP 2002) OPAL: Euro. Phys. J. C23, 397 (2002)

[4] ALEPH: Phys. Lett. B543, 1 (2002) DELPHI: 2002-038-CONF-572 (ICHEP 2002) L3: Note 2686 (EPS 2001) OPAL: Physics Note \#509 (ICHEP 2002) LEP combination: hep-ex/0107031

[5] DELPHI: Phys. Lett. B552, 127 (2003) OPAL: Phys. Lett. B526, 221 (2002)

[6] DELPHI: 2002-035-CONF-569 OPAL: Physics Note \#507 (ICHEP 2002) LEP combination: LHWG Note/2001-07

[7] OPAL: CERN-EP-2002-032

[8] OPAL: CERN-EP/2002-058

[9] L3 note \#2774 (ICHEP2002)

[10] OPAL: Euro. Phys. J. C18, 425 (2201) and Physics Note \#475 